\title{
Comparação de métodos de estimação da estabilidade fenotípica em animais da raça Holandesa ${ }^{(1)}$
}

\author{
Douglas Messias Lamounier Camargos Rezende ${ }^{(2)}$ e Daniel Furtado Ferreira ${ }^{(3)}$
}

\begin{abstract}
Resumo - Este trabalho teve por objetivo comparar os métodos de regressão convencional, desvio do desempenho máximo, índice de risco e o modelo AMMI (Additive Multiplicative Models Interaction) na estimação de parâmetros de estabilidade de leite de vaca da raça Holandesa. Os resultados obtidos foram comparados com os do método de Toler. Foram utilizados registros de 22.560 lactações em até 305 dias, obtidos na Associação de Criadores de Gado Holandês de Minas Gerais (ACGHMG), entre os anos de 1989 e 1996. Os animais foram separados em seis grupos genéticos e submetidos a 14 ambientes. Os métodos de regressão convencionais apresentaram classificações, com relação às variações ambientais, muito diferentes da classificação do método de Toler; este último foi considerado mais adequado. O método AMMI não foi eficiente para estudar a estabilidade fenotípica dos grupamentos genéticos da raça Holandesa. Os métodos do desvio do desempenho máximo e do índice de risco apresentaram resultados semelhantes entre si e complementaram as informações fornecidas pelo método de Toler. Os grupamentos GC2 (segunda geração controlada) e PO (puro de origem) foram os que apresentaram as maiores produtividades médias, e se alternaram entre si como os mais estáveis nos diferentes métodos. Os grupamentos 31/32 e GC1 foram os de pior desempenho médio, e estão sempre entre os mais instáveis em todos os métodos.
\end{abstract}

Termos para indexação: leite, rendimento, métodos de análise.

\section{Comparison of methods to estimate the phenotype stability in Holstein dairy cattle breed}

\begin{abstract}
This work aimed to compare conventional regression methods, deviation from the maximum response, reliability index and AMMI (Additive Multiplicative Models Interaction) in order to estimate stability parameters for milk production in Holstein cows. The results obtained were compared with the results from Toler's method. In total 22,560 lactation data were used, up to 305 days obtained through the Holstein Cattle Breeders Association of Minas Gerais State (HCBAMG), Brazil,

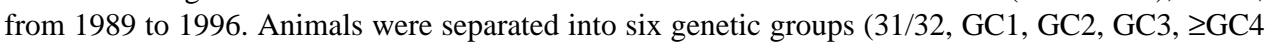
and $\mathrm{PO}$ ) and evaluated in 14 environments. The conventional regression method presented classification patterns for environmental variations different from the classification given by Toler's method. The latter was considered more appropriate. The AMMI method was not considered to be efficient for the study of phenotypic stability of the Holstein breed groups. The methods of deviation from the maximum response and reliability index presented similar results and complemented information supplied by the method of Toler. The genetic groups GC2 $\left(2^{\text {nd }}\right.$ controlled generation $)$ and PO (pure from origin) presented the largest average productions and alternated with each other as the most stable for the different methods. The genetic groups $31 / 32$ and GC1 presented the lowest average productions and were consistently the most unstable for all the methods studied.
\end{abstract}

Index terms: milk, yields, analytical methods.

(1) Aceito para publicação em 9 de março de 2000.

(2) Rua Antônio Gomes Macedo, 51, Bairro Nações, CEP 35595-000 Luz, MG. E-mail: dougmes@yahoo.com.br

(3)Universidade Federal de Lavras, Dep. de Ciências Exatas, Caixa Postal 37, CEP 37200-000 Lavras, MG. Bolsista CNPq. E-mail: danielff@ufla.br

\section{Introdução}

Todos os estudos com animais, que tratam da interação genótipo $\mathrm{x}$ ambiente, utilizam o resultado da interação touro x região ou o coeficiente de herdabilidade (Rorato et al., 1994) para expressar esta 
interação. Isto se torna muito dispendioso, pois seria necessário testar todos os animais ou o sêmen importado para verificar sua superioridade também nas condições brasileiras.

Uma alternativa interessante seria utilizar os métodos desenvolvidos em plantas para obtenção da interação genótipo $\mathrm{x}$ ambiente e tentar adaptá-los para que possam ser utilizados em animais. Muitos métodos foram desenvolvidos e discutidos na literatura com a finalidade de avaliar o padrão de desempenho genotípico nestes ambientes e vêm sendo largamente aplicados ao melhoramento de plantas (Lin et al., 1986; Westcott, 1986; Becker \& Leon, 1988; Crossa, 1990).

O único estudo, no País, em que foram encontrados relatos do uso de tal método aplicado ao melhoramento animal foi realizado por Rezende et al. (1999), utilizando o método de Toler (1990) para constatar a estabilidade fenotípica em animais de diferentes grupamentos genéticos da raça Holandesa no Estado de Minas Gerais.

Experimentos para avaliar a interação genótipo $\mathrm{x}$ ambiente, apesar de sua importância, não fornecem informações pormenorizadas sobre o comportamento de cada genótipo em face das variações ambientais. Estratégias que permitem identificar os genótipos de comportamento previsível e que respondem positivamente às variações ambientais favoráveis são denominadas de análises de adaptabilidade e estabilidade (Plaisted \& Paterson, 1959; Finlay \& Wilkinson, 1963; Wricke, 1964; Eberhart \& Russell, 1966; Tai, 1971; Verma et al., 1978; Cruz et al., 1989).

Um método bastante utilizado no melhoramento de plantas é o do desvio do desempenho máximo (Lin \& Binns, 1988). Os resultados de estabilidade obtidos por este método são baseados tanto no efeito médio genotípico, como no efeito da interação genótipo x ambiente. Cada genótipo é comparado com a resposta máxima em cada ambiente, fornecendo uma medida de superioridade. Essa medida $\left(\mathrm{P}_{\mathrm{i}}\right)$ do genótipo é definida por Lin \& Binns (1988) como a distância entre o quadrado médio do genótipo i e o genótipo com resposta máxima.

O índice de risco de cada genótipo é um parâmetro de avaliação da estabilidade fenotípica proposto por Annicchiarico (1992). Os autores propõem que se considere um coeficiente de confiança de $75 \%$, e que as médias genotípicas sejam expressas em termos da porcentagem dos valores médios dos ambientes, e que, em seguida, se estime a média e o desvio-padrão de cada genótipo em relação aos ambientes. E de posse desses valores, e adotando-se a distribuição normal, se estimem os índices de risco, que fornecem a idéia, fixando uma probabilidade de confiança, da proporção mínima, que um determinado genótipo supera a média dos ambientes estudados. Quanto maior o valor do índice de risco, mais estável será considerado o genótipo.

Destaca-se também o Método Multivariado AMMI (Additive Multiplicative Models Interaction) (Gauch Junior \& Zobel, 1988; Crossa, 1990; Yau, 1995), que vem sendo largamente utilizado. Esse método baseia-se inicialmente na estimação de efeitos aditivos dos genótipos e dos ambientes, pelo procedimento usual da análise de variância (ANAVA). Em seguida, são estimados os efeitos da interação genótipo $\mathrm{x}$ ambiente, considerados multiplicativos, utilizando a análise dos componentes principais (ACP), de acordo com Crossa (1990).

Outro método, que avalia o padrão de comportamento genotípico em face do ambiente através de um modelo e teste que mantém o rigor estatístico exigido nas aproximações por regressão, foi apresentado por Toler (1990). Trata-se de um modelo não-linear nos parâmetros, apresentado nas versões de regressão linear simples, análogo ao modelo de Eberhart \& Russell (1966), e de regressão linear bissegmentado, análogo ao modelo de Cruz et al. (1989). Esses modelos são utilizados juntamente com o desempenho médio de cada genótipo, para agrupálos de acordo com o seu padrão de resposta ao ambiente. Este método fundamenta-se basicamente no fato de que o índice ambiental é um parâmetro a ser estimado e que os modelos são não-lineares nos parâmetros, exigindo métodos mais refinados de estimação, como, por exemplo, o método de estimação de Gauss Newton, modificado (Gallant, 1987).

O objetivo deste trabalho foi comparar os métodos de regressão convencional, desvio do desempenho máximo, índice de risco e do modelo AMMI (Additive Multiplicative Models Interaction) na estimação de parâmetros de estabilidade da produtividade de leite de vaca da raça Holandesa. 


\section{Material e Métodos}

Foram utilizados os dados de produção de leite de 22.560 lactações, obtidos na Associação de Criadores de Gado Holandês de Minas Gerais (ACGHMG), entre os anos de 1989 e 1996 . Os animais foram representados por seis grupamentos genéticos: 31/32, GC1 (primeira geração controlada), GC2, GC3, agrupamento das demais gerações controladas, denominado $\geq \mathrm{GC} 4$ (GC4 a GC11), e PO (puro de origem). Cada grupo foi submetido a 14 ambientes (combinações entre sete núcleos de criadores de gado Holandês do Estado de Minas Gerais e dois níveis de manejo).

Foi usada a característica de produção de leite em até 305 dias. Os registros de animais com duração de lactação inferior a 150 dias e com produção menor que $750 \mathrm{~kg}$ foram eliminados das análises. Quando a lactação se estendeu por mais de 305 dias, considerou-se a produção obtida até 305 dias. Foram utilizados somente animais cujas produções foram obtidas em duas ordenhas. Animais que apresentaram causas de secagem anormais foram eliminados.

Foram consideradas duas estações de parição: a estação seca e a estação das águas. A estação seca foi definida pelos meses de abril a setembro, enquanto a estação das águas foi definida pelos meses de outubro a março.

Os métodos de análises empregados para estimação dos parâmetros de estabilidade foram o de regressão (Eberhart \& Russell, 1966; Cruz et al., 1989), o de desvio do desempenho máximo (Lin \& Binns, 1988), o de índice de risco (Annicchiarico, 1992) e o AMMI (Gauch Junior \& Zobel, 1988). Os modelos de Toler (1990) foram ajustados pelos quadrados mínimos aplicados a modelos não-lineares nos parâmetros. O modelo 1 de Toler, que prevê um comportamento linear dos genótipos em face da variação ambiental é dado por:

$Y_{i j}=\alpha_{i}+\beta_{i} \mu_{j}+\delta_{i j}+\varepsilon_{i j}$

em que, $Y_{\mathrm{ij}}$ é a média de produção do i-ésimo genótipo no j-ésimo ambiente; $\alpha_{i}$ é a média de produção do i-ésimo genótipo; $\beta_{\mathrm{i}}$ é o coeficiente de sensibilidade de resposta ao ambiente do i-ésimo genótipo; $\mu_{\mathrm{j}}$ representa o índice ambiental; $\delta_{\mathrm{ij}}$ é o desvio de regressão do i-ésimo genótipo no j-ésimo ambiente; $\varepsilon_{\mathrm{ij}}$ é o efeito do erro médio associado à observação $Y_{i j} ; i=1,2, \ldots, p ; j=1,2, \ldots, q$. A única diferença deste modelo em relação ao de Eberhart \& Russell (1966) é que o índice ambiental $\mu_{\mathrm{j}}$ é um parâmetro a ser estimado, juntamente com os $\alpha_{i}$ 's e $\beta_{\mathrm{i}}$ 's., sujeitos às restrições $\sum_{\mathrm{i}=1}^{\mathrm{p}} \beta_{\mathrm{i}}=\mathrm{p}$ e $\sum_{\mathrm{j}=1}^{\mathrm{q}} \mu_{\mathrm{j}}=0$.

O modelo 2 de Toler (1990), similar ao de Cruz et al. (1989), é apresentado a seguir:

$$
Y_{i j}=\alpha_{i}+\left[\beta_{1 i} Z_{j}+\beta_{2 i}\left(1-Z_{j}\right)\right]+\delta_{i j}+\varepsilon_{i j},
$$

em que $\mathrm{Y}_{\mathrm{ij}}$ é a média de produção do i-ésimo genótipo no j-ésimo ambiente; $\alpha_{i}$ representa o intercepto de resposta do i-ésimo genótipo; $\beta_{1 \mathrm{i}}$ e $\beta_{2 \mathrm{i}}$ representam os coeficientes de sensibilidade de resposta do i-ésimo genótipo em ambientes desfavorável e favorável, respectivamente; $\mu_{\mathrm{j}}$ representa o efeito ambiental; $\delta_{\mathrm{ij}}$ é o desvio de regressão do i-ésimo genótipo no j-ésimo ambiente; $\varepsilon_{\mathrm{ij}}$ é o efeito do erro médio associado à observação $Y_{i j} ; i=1,2, \ldots, p$; $\mathrm{j}=1,2, \ldots, \mathrm{q} ; \mathrm{Z}_{\mathrm{j}}=1$ se $\mu_{\mathrm{j}} \leq 0$, e $\mathrm{Z}_{\mathrm{j}}=0$ se $\mu_{\mathrm{j}}>0$. As restrições impostas a este modelo são: $\sum_{i=1}^{p} \beta_{1 i}=\sum_{i=1}^{p} \beta_{2 i}=p$ e $\sum_{j=1}^{q} \mu_{j}=0$. Os genótipos foram classificados de acordo com estes modelos quanto aos seus padrões de resposta ao ambiente. Essa classificação pode ser resumida da seguinte forma: A - duplamente desejável, ou seja, genótipos podem ser adequados tanto para ambientes de alta quanto de baixa qualidade; B - desejável somente para ambientes de alta qualidade; C - resposta linear média; D - desejável somente para ambientes de baixa qualidade; E - duplamente indesejável.

Em seguida, utilizou-se o método de Lin \& Binns (1988). A justificativa de utilizar tal método é o fato de a classificação de Toler (1990) representar padrões de comportamento em face da variação ambiental, mas que não representam uma medida da estabilidade fenotípica, uma vez que os desvios de regressão não são isolados de cada genótipo individualmente. Foram estimados os valores de P(i) (índice de estabilidade do genótipo i), que se referem a uma soma de quadrados do componente da interação, tomando-se como referência o desempenho máximo de todos os genótipos em cada ambiente. Essas estimativas permitiram que se testassem as hipóteses de nulidade do valor paramétrico de $\mathrm{P}(\mathrm{i})$, com base em um teste $\mathrm{F}$. A significância dessa hipótese indicaria que o genótipo difere estatisticamente do máximo, e um P(i) nulo indicaria que ele tem desempenho semelhante ao máximo ao longo dos ambientes. Essas estimativas foram decompostas em um efeito genético e um desvio atribuído à interação.

Visando obter mais informações sobre a estabilidade dos genótipos, foi aplicado o método proposto por Annicchiarico (1992). Neste método estimou-se o índice de risco de cada genótipo, I(i), considerando um coeficiente de confiança de $75 \%$. As médias dos genótipos foram expressas em termos da porcentagem dos valores médios dos ambientes, e em seguida estimou-se a média e o desvio-padrão de cada grupamento genético em relação aos ambientes. Com estes valores, e com a distribuição normal, foram estimados os índices de risco de cada genótipo. 
Em seguida, foi utilizado o método de análise cujos efeitos principais (genótipos e ambientes) são aditivos e o efeito da interação é multiplicativo (modelo AMMI). Buscou-se avaliar a viabilidade da utilização da técnica multivariada AMMI, para estudos de estabilidade fenotípica em animais.

Os resultados obtidos pelos diferentes métodos foram comparados entre si e com os resultados obtidos por Rezende et al. (1999), que utilizaram os dois modelos de Toler (1990). Foram identificados os métodos mais apropriados a serem usados em pesquisas sobre estabilidade fenotípica em animais.

\section{Resultados e Discussão}

Na Tabela 1 são apresentados os resultados da utilização do modelo de Cruz et al. (1989) relativos ao teste da hipótese $\mathrm{H}_{0}: \beta_{2}=0$, no que tange aos grupamentos genéticos avaliados no presente estudo. Os resultados mostram que se deve rejeitar a hipótese nula, $\beta_{2}=0$, apenas no que diz respeito ao genótipo GC2. Esse resultado permite inferir que este genótipo apresenta um comportamento que se enquadra no modelo bissegmentado e que os demais genótipos se enquadram no modelo linear de resposta ao ambiente.

Na Tabela 2 são apresentadas as estimativas dos parâmetros $\alpha$ e $\beta$ do modelo de Eberhart \& Russell (1966), e o teste para $\mathrm{H}_{0}: \beta=1$. O parâmetro $\alpha$ representa a produtividade média dos diferentes genótipos, onde se nota que o genótipo PO é o de maior produtividade, enquanto os genótipos 31/32 e GC1 são os de menor produtividade. Por meio do teste da $\mathrm{H}_{0}: \beta=1$ pode-se inferir que a produção leiteira dos genótipos 31/32 e GC3 não apresenta respostas positivas a melhorias ambientais, e os animais são avaliados como pertencentes à classe $\mathrm{D}$, segundo a classificação de Toler (1990). Os genótipos GC1 e $\geq$ GC4 apresentam resposta média à mudança ambiental, sendo classificados como pertencentes à classe $\mathrm{C}$. $\mathrm{O}$ genótipo $\mathrm{PO}$ apresentou $\beta>1$, e é considerado responsivo às melhorias ambientais. Por ter apresentado a maior produtividade média e por ter sido classificado como pertencente à classe $\mathrm{B}$, pode ser indicado para ambientes de alta qualidade. A classificação alcançada por este genótipo mostra que ele
Tabela 1. Estimativas de $\beta_{2}$ obtidas pelo método de Cruz et al. (1989) e teste da hipótese $\mathrm{H}_{0}: \beta_{2}=0$, para confirmar a necessidade do uso ou não de modelos bissegmentados, em análises de estabilidade de produtividade de leite em genótipos de gado holandês.

\begin{tabular}{lcc}
\hline Genótipo $_{(\mathrm{i})}^{(1)}$ & $\beta_{2(\mathrm{i})}^{(2)}$ & $\mathrm{t}\left(\mathrm{H}_{0}: \beta_{2(\mathrm{i})}=0\right)$ \\
\hline $31 / 32$ & $-0,1855$ & $-0,5561^{\mathrm{ns}}$ \\
$\mathrm{GC} 1$ & $-0,3360$ & $-1,0072^{\mathrm{ns}}$ \\
$\mathrm{GC} 2$ & 0,7208 & $2,1605^{*}$ \\
$\mathrm{GC} 3$ & 0,6283 & $1,8833^{\mathrm{ns}}$ \\
$\geq \mathrm{GC} 4$ & $-0,2064$ & $-0,6187^{\mathrm{ns}}$ \\
PO & $-0,6211$ & $-1,8617^{\mathrm{ns}}$ \\
\hline
\end{tabular}

(1) $\mathrm{i}=1,2, \ldots, 6$. (2) Erro-padrão de $\beta_{2}: 0,3336$. ns Não-significativo. * Significativo a $5 \%$ de probabilidade pelo teste t.

Tabela 2. Estimativas dos coeficientes angular $(\alpha)$ e intercepto ( $\beta$ ) no modelo de Eberhart \& Russell (1966), bem como do teste t para a hipótese $\mathrm{H}_{0}: \beta=1$, em análises de estabilidade de produtividade de leite em genótipos de gado holandês.

\begin{tabular}{lccc}
\hline Genótipo $_{(\mathrm{i})}$ & $\alpha_{(\mathrm{i})}(\text { média })^{(1)}$ & $\beta_{(\mathrm{i})}{ }^{(2)}$ & $\mathrm{t}\left(\mathrm{H}_{0}: \beta_{(\mathrm{i})}=1\right)$ \\
\hline $31 / 32$ & $4.713,9$ & 0,7958 & $-2,0355^{*}$ \\
$\mathrm{GC} 1$ & $4.973,5$ & 0,9493 & $-0,5052^{\text {ns }}$ \\
$\mathrm{GC} 3$ & $5.269,0$ & 0,7899 & $-2,0938^{*}$ \\
$\geq \mathrm{GC} 4$ & $5.325,3$ & 1,0583 & $0,5806^{\text {ns }}$ \\
PO & $5.509,4$ & 1,2943 & $2,9331^{* *}$ \\
\hline
\end{tabular}

(1) Erro-padrão de $\alpha: 70,59$. (2) Erro-padrão de $\beta: 0,1003$. ns Não-significativo. ${ }^{*}$ Significativo a $5 \%$ de probabilidade pelo teste $\mathrm{t} .{ }^{* *}$ Significativo a $1 \%$ de probabilidade pelo teste $t$.

possui um elevado risco de apresentar baixas produtividades caso seja recomendado para ambientes de baixa qualidade.

Quanto ao genótipo GC2 (segunda geração controlada), que foi o único a apresentar o teste significativo para $\mathrm{H}_{0}$ : $\beta_{2}=0$, foram aplicados nele os testes para as hipóteses $\mathrm{H}_{0}: \beta_{1}=1$ e $\mathrm{H}_{0}: \beta_{1}+\beta_{2}=1$. $\mathrm{O}$ teste da $\mathrm{H}_{0}: \beta_{1}=1$ foi não-significativo $(\mathrm{t}=0,38)$, ou seja, a hipótese nula não deve ser rejeitada, o que indica que o genótipo GC2 apresenta resposta média em termo de produção em ambientes desfavoráveis ( $\left.\hat{\beta}_{1}=1,04\right)$. A hipótese $H_{0}: \beta_{1}+\beta_{2}=1$ foi rejeitada $(p<0,05 ; t=2,40)$, e conforme a estimativa obtida $\left(\hat{\beta}_{1}+\hat{\beta}_{2}\right)$ pode-se inferir que o genótipo em questão foi responsivo à melhoria ambiental em ambientes favoráveis. Nenhuma classificação dessa na- 
tureza foi proposta por Toler (1990). No entanto, esse genótipo foi classificado como pertencente à classe I, ou seja, de resposta média a ambientes desfavoráveis e responsivo a ambientes favoráveis.

Os resultados referentes à classificação obtida no presente trabalho foram: 31/32 (D), GC1 (C),

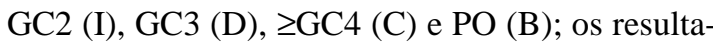
dos referentes à classificação obtida por Rezende et al. (1999) a partir dos modelos de Toler (1990) foram: 31/32 (D), GC1 (C), GC2 (A), GC3 (F), $\geq \mathrm{GC} 4$ (E) e PO (G). Pode-se notar diferenças nas classificações obtidas nos dois métodos. É conveniente salientar que as classificações obtidas a partir dos modelos tradicionais estão sujeitas a erros, uma vez que os índices ambientais não são independentes da variável resposta. O método de Toler (1990), por considerar os índices como parâmetros a serem estimados, elimina essa principal desvantagem dos métodos clássicos, e portanto, seu resultado é considerado mais adequado.

Nota-se que o genótipo GC1 foi o único que apresentou o mesmo resultado nos dois métodos. O genótipo PO, classificado pelo método de Toler (1990) como de resposta média a ambientes favoráveis, e que não deve ser recomendado para ambientes de baixa qualidade, passou a ter resposta linear $\operatorname{com} \beta>1$, ou seja, a responder à melhoria ambiental, mas ainda não é apropriado para recomendação em ambientes desfavoráveis. O GC3, adaptado em condições desfavoráveis $\left(\beta_{1}<1\right)$ e com resposta média à melhoria ambiental $\left(\beta_{2}=1\right)$, passou a ter classificação $\mathrm{D}(\beta<1)$, o que o tornaria inadequado para ambientes de alta qualidade. $\mathrm{O}$ genótipo $\geq \mathrm{GC} 4$, considerado como duplamente indesejável, passou a ter resposta média aos ambientes. Finalmente, o GC2, considerado duplamente favorável, passou a ter resposta média a ambientes desfavoráveis. Tomando-se o método de Toler (1990) como mais adequado, por eliminar a desvantagem da dependência dos índices ambientais das médias genotípicas, verificou-se que a classificação a partir dos modelos clássicos de regressão levou a diferentes resultados de classificação, na grande maioria. Isso mostra que os refinamentos metodológicos propostos têm sido benéficos para os melhoristas.
Na Tabela 3 são apresentados os resultados dos componentes principais, do método AMMI, os autovalores, e a porcentagem da explicação dada por eles. Verificou-se que a explicação dada pelo primeiro componente $(41,17 \%)$ não foi suficiente para atingir os $80 \%$ ou mais da variação da interação explicada pelos componentes principais, que é o ideal para este método (Yau, 1995; Arias, 1996). Para atingir este valor, foi necessária a utilização dos três primeiros componentes $(83,43 \%)$, o que não é conveniente quando se utiliza o método AMMI, o ideal é a explicação máxima possível no primeiro componente principal, para que a interpretação dos resultados seja facilitada e mais concisa. Isso porque com três dimensões, as interpretações e representações geométricas não são triviais.

Na Tabela 4 apresenta-se o resumo da análise de variância com a decomposição das somas dos quadrados da interação genótipo x ambiente (modelo AMMI). Verificou-se que os dois primeiros componentes principais foram altamente significativos $(\mathrm{P}<0,01)$; o terceiro, significativo $(\mathrm{P}<0,05)$; e os desvios, não-significativos, o que demonstra que os três primeiros componentes são necessários para explicar com precisão a variação da interação, e, como já comentado, inviabiliza a utilização prática. $\mathrm{O}$ uso do método AMMI é recomendado quando o primeiro componente principal explica no mínimo $80 \%$ da variação da interação.

Na Tabela 5 são apresentados os resultados rela-

Tabela 3. Resultados da análise considerando o modelo com efeitos principais aditivos e interação multiplicativa (AMMI), com estimação dos componentes principais (CP) e a sua correspondente porcentagem da explicação da interação, em análises de estabilidade de produtividade de leite em genótipos de gado holandês.

\begin{tabular}{crcc}
\hline CP & Autovalores & $\begin{array}{c}\text { Explicação } \\
(\%)\end{array}$ & $\begin{array}{c}\text { Explicação } \\
\text { acumulada (\%) }\end{array}$ \\
\hline 1 & $4.171 .571,1$ & 41,17 & 41,17 \\
2 & $2.640 .240,9$ & 26,04 & 67,21 \\
3 & $1.643 .157,7$ & 16,22 & 83,43 \\
4 & $1.009 .458,4$ & 9,96 & 93,39 \\
5 & $669.453,0$ & 6,61 & 100,00 \\
\hline
\end{tabular}

Pesq. agropec. bras., Brasília, v. 36, n. 1, p. 179-186, jan. 2001 
tivos ao modelo de Lin \& Binns (1988), e o teste de Scott-Knott relativo às produtividades médias. Segundo o teste de Scott \& Knott (1974), as médias de produção dos genótipos GC2, GC3, \GC4 e PO não diferem estatisticamente entre si, e são consideradas superiores às médias dos demais genótipos. O pior desempenho foi o do genótipo 31/32, com média de produção de 4.713,87 kg de leite em até 305 dias.

O método de Lin \& Binns (1988) considera o desvio $\left(\mathrm{P}_{\mathrm{i}}\right)$ em relação ao desempenho máximo em cada local. Observa-se que o PO apresentou melhor desempenho, não diferiu significativamente do máximo; foi considerado o mais estável, e participou com a menor porcentagem para a interação. O genótipo $\mathrm{GC} 2$, que foi considerado por outros modelos como

Tabela 4. Resumo da análise de variância com a decomposição das somas dos quadrados da interação genótipo $\mathrm{x}$ ambiente (modelo AMMI), em análises de estabilidade de produtividade de leite em genótipos de gado holandês.

\begin{tabular}{lcc}
\hline Fonte de variação & GL & QM \\
\hline Genótipos (G) & 5 & $36.525 .441,7^{* *}$ \\
Ambientes (A) & 13 & $94.222 .603,0^{* *}$ \\
G x A & $(65)$ & $4.592 .172,9^{* *}$ \\
$\mathrm{CP}-1$ & 17 & $7.227 .806,4^{* *}$ \\
$\mathrm{CP}-2$ & 15 & $5.184 .514,2^{* *}$ \\
$\mathrm{CP}-3$ & 13 & $3.722 .988,4^{*}$ \\
Desvios & 20 & $2.472 .598,2^{\text {ns }}$ \\
Resíduo & 22.461 & $2.055 .000,0$ \\
\hline
\end{tabular}

ns Não-significativo. * Significativo a $5 \%$ de probabilidade pelo teste $\mathrm{F}$. ** Significativo a $1 \%$ de probabilidade pelo teste $\mathrm{F}$. o mais estável, ficou em segundo lugar e bem próximo do PO, embora tenha apresentado $\mathrm{P}_{\mathrm{i}}$ significativamente diferente do máximo. Os genótipos de maior $\mathrm{P}_{\mathrm{i}}$ foram o 31/32 e o GC1, o que concorda com as demais análises. O material mais instável, ou o que mais contribuiu para a interação, foi o GC3, o qual foi considerado o de pior desempenho.

$\mathrm{Na}$ Tabela 6 são apresentados os resultados da utilização do método de análise de Annicchiarico (1992). Neste método também se destacou o genótipo PO, que apresenta $75 \%$ de confiança de, na pior das hipóteses, apresentar produtividade média 1,49\% acima da média dos ambientes. Também neste modelo o GC2 esteve próximo ao melhor desempenho. Os genótipos 31/32 e GC1, de acordo com todos os resultados dos demais métodos, foram os de pior desempenho.

Em razão de limitações nas bases teóricas de alguns métodos, ou ao não-atendimento às exigências mínimas requeridas por outros para a obtenção da eficiência preconizada, os resultados mostraram-se não-consistentes. Os modelos de regressão de Cruz et al. (1989) e os de Eberhart \& Russell (1966) e o modelo AMMI se enquadram, respectivamente, nestes casos. Por outro lado, fica evidenciado que alguns dos diferentes métodos de análise da estabilidade fenotípica fornecem resultados que se complementam, como é o caso dos métodos de Lin \& Binns (1988) e Annicchiarico (1992) com relação aos modelos de regressão propostos por Toler (1990).

Tabela 5. Análise de estabilidade utilizando o método de Lin \& Binns (1988), apresentando as estimativas de $\mathrm{P}_{(\mathrm{i})}$ e da sua decomposição em efeito genético e desvio da interação, e comparação das médias genotípicas pelo teste de Scott \& Knott (1974), em análises de estabilidade de produtividade de leite em genótipos de gado holandês.

\begin{tabular}{lcrrrr}
\hline Genótipo & Médias $^{(1)}$ & $\mathrm{P}_{(\mathrm{i})}$ & Efeito genético & $\begin{array}{c}\text { Desvio da } \\
\text { interação }\end{array}$ & $\begin{array}{c}\text { Contribuição para } \\
\text { a interação }(\%)\end{array}$ \\
\hline $31 / 32$ & $4.713,9 \mathrm{c}$ & $18.072 .388,4^{*}$ & $15.625 .338,5$ & $2.447 .050,0$ & 13,30 \\
$\mathrm{GC} 1$ & $4.973,5 \mathrm{~b}$ & $13.095 .918,5^{*}$ & $8.740 .901,7$ & $4.355 .016,8$ & 23,67 \\
$\mathrm{GC} 2$ & $5.397,7 \mathrm{a}$ & $4.272 .237,5^{*}$ & $1.765 .644,1$ & $2.506 .593,4$ & 13,63 \\
GC3 & $5.269,0 \mathrm{a}$ & $8.424 .452,3^{*}$ & $3.320 .900,7$ & $5.103 .551,6$ & 27,74 \\
$\geq$ GC4 & $5.325,3 \mathrm{a}$ & $5.449 .835,6^{*}$ & $2.580 .374,4$ & $2.869 .461,3$ & 15,60 \\
PO & $5.509,4 \mathrm{a}$ & $1.924 .803,9$ & $809.837,6$ & $1.114 .966,3$ & 6,06 \\
\hline
\end{tabular}

${ }^{(1)}$ Médias seguidas da mesma letra são estatisticamente iguais segundo o teste Scott \& Knott (1974). * Difere significativamente do máximo (P<0,05); ponto de corte $($ Cutoff Point $)=3.535 .816,0$. 
Tabela 6. Análise de estabilidade da produtividade de leite em genótipos de gado holandês, de acordo com o método de Annicchiarico (1992), apresentando o índice de risco $\left(\mathrm{I}_{(\mathrm{i})}\right)$, considerando-se $75 \%$ de confiança.

\begin{tabular}{lccr}
\hline Genótipo & Média (\%) & Desvio-padrão (\%) & $\mathrm{I}_{(\mathrm{i})}{ }^{(1)}$ \\
\hline $31 / 32$ & 90,88 & 5,02 & 87,50 \\
GC1 & 95,70 & 5,85 & 91,75 \\
GC2 & 103,75 & 6,87 & 99,12 \\
GC3 & 101,79 & 8,90 & 95,79 \\
$\geq$ GC4 & 102,34 & 7,58 & 97,23 \\
PO & 105,54 & 6,01 & 101,49 \\
\hline
\end{tabular}

(1) Nível de significância adotado $=0,25$.

\section{Conclusões}

1. O método AMMI não é eficiente para estudar a estabilidade fenotípica dos grupamentos genéticos da raça Holandesa, por ter tido uma explicação do modelo no primeiro componente de apenas $41,17 \%$.

2. Os melhores métodos em termos de análise de estabilidade fenotípica são os de Lin \& Binns, de Annicchiarico e de Toler; devem ser aplicados concomitantemente, por se complementarem mutuamente.

3. Os grupamentos genéticos GC2 e PO são os que apresentam as maiores produtividades médias e se alternam entre si como os mais estáveis nos diferentes métodos, o que mostra que a maior estabilidade genotípica está associada a maiores produtividades de leite.

4. Os grupamentos genéticos 31/32 e GC1 são os de pior desempenho médio e os mais instáveis, o que mostra uma associação inversa entre a estabilidade fenotípica e a produtividade.

\section{Referências}

ANNICCHIARICO, P. Cultivar adaptation and recommendation from alfalfa trials in Northern Italy. Journal of Genetics \& Breeding, Rome, v. 46, n. 1, p. 269-278, Mar. 1992.

ARIAS, E. R. A. Adaptabilidade e estabilidade dos cultivares de milho avaliados no Estado de Mato Grosso do Sul e avanço genético obtido no período de 1986/87 a 1993/94. Lavras : UFLA, 1996. 118 p. Tese de Doutorado.

BECKER, H. C.; LEON, J. Stability analysis in plant breeding. Plant Breeding, Berlin, v. 101, n. 1, p. 1-23, 1988.
CROSSA, J. Statistical analysis of multilocations trials. Advances in Agronomy, San Diego, v. 44, p. 55-85, 1990.

CRUZ, C. D.; TORRES, R. A. de; VENCOVSKY, R. An alternative approach to the stability analysis proposed by Silva and Barreto. Revista Brasileira de Genética, Ribeirão Preto, v. 12, n. 2, p. 567-580, 1989.

EBERHART, S. A.; RUSSELL, W. A. Stability parameters for comparing varieties. Crop Science, Madison, v. 6, n. 1, p. 36-40, 1966.

FINLAY, K. W.; WILKINSON, G. N. The analysis of adaptation in a plant breeding program. Australian Journal of Agriculture Research, Collingwood, v. 14, n. 6, p. 742-754, 1963.

GALLANT, A. R. Nonlinear statistical models. New York : J. Wiley, 1987. $610 \mathrm{p}$.

GAUCH JUNIOR, H. G.; ZOBEL, R. W. Predictive and postdictive success of statistical analysis of yield trials. Theoretical and Applied Genetics, Berlin, v. 76, n. 1, p. 1-10, 1988.

LIN, C. S.; BINNS, M. R. A superiority measure of cultivar performance for cultivar x location data. Canadian Journal of Plant Science, Ottawa, v. 68, n. 1, p. 193-198, Jan. 1988.

LIN, C. S.; BINNS, M. R.; LEFKOVICTH, L. P. Stability analysis: where do we stand? Crop Science, Madison, v. 26, n. 5, p. 894-900, 1986.

PLAISTED, R. L.; PATERSON, L. C. A technique for evaluating the ability of selections to yield consistently in different locations or seasons. American Potato Journal, Orono, v. 36, n. 11, p. 381-385, 1959.

REZENDE, D. M. L. C.; FERREIRA, D. F.; GONÇALVES, T. M. Avaliação da estabilidade fenotípica para produção de leite em animais da raça Holandesa. Revista Brasileira de Zootecnia, Viçosa, v. 28, n. 4, p. 735-742, 1999.

RORATO, P. R. N.; LÔBO, R. B.; MARTINS FILHO, R.; RIBAS, N. P. Efeito da interação genótipo $x$ ambiente sobre a produção de leite da raça Holandesa, no Estado do Paraná. Revista Brasileira de Zootecnia, Viçosa, v. 23, n. 5, p. 859-869, 1994.

SCOTT, A. J.; KNOTT, M. A cluster analysis method for grouping means in the analysis of variance. Biometrics, Washington, v. 30, p. 507-512, Sept. 1974. 
TAI, J. G. C. Genotype stability analysis and its application to potato regional trials. Crop Science, Madison, v. 11, n. 2, p. 184-190, 1971.

TOLER, J. E. Patterns of genotypic performance over environmental arrays. Raleigh : Clemson University, 1990. 154 p. Ph.D. Thesis.

VERMA, M. M.; CHAHAL, G. S.; MURTY, B. R. Limitations of conventional regression analysis: a proposed modification. Theoretical and Applied Genetics, Berlin, v. 53, p. 89-91, 1978.
WESTCOTT, B. Some methods of analyzing genotype-environment interactions. Heredity, Oxford, v. 56, p. 243-253, 1986.

WRICKE, G. Zur Berechnung der Ökovalenz bei Sommerweizen und Hafer. Zeitschrift fuer Pflanzenzuechtung, Berlin, v. 52, p. 127-138, 1964.

YAU, S. K. Regression and AMMI analysis of genotype $\mathrm{x}$ environmental interactions: an empirical comparison. Agronomy Journal, Madison, v. 87, n. 1, p. 121-126, 1995. 